DEPÓSITO LEGAL ZU2020000153

Esta publicación científica en formato digital

es continuidad de la revista impresa

ISSN 0041-8811

E-ISSN 2665-0428

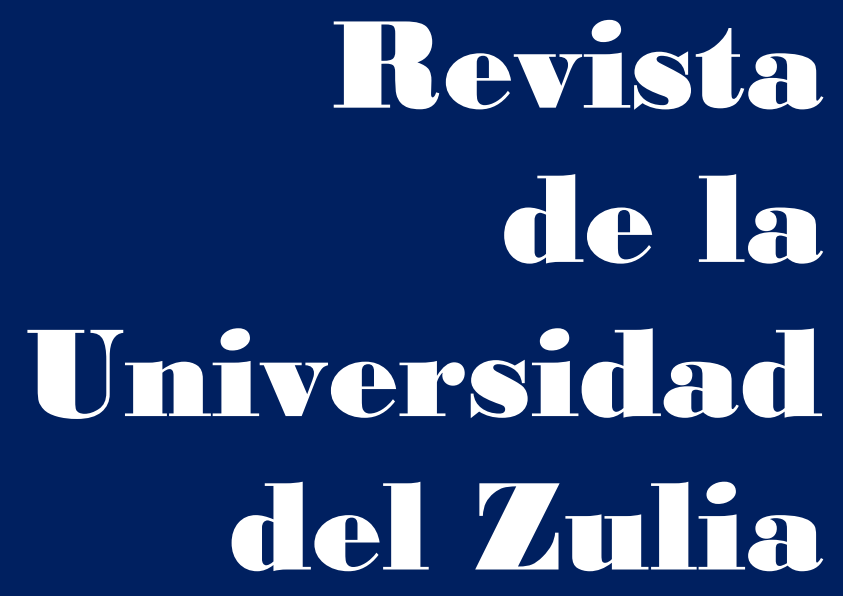

Fundada en 1947

por el Dr. Jesús Emrique Lossada

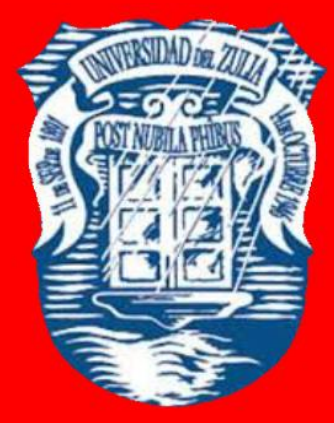

Ciencias

Sociales

y Arte

Año $12 \quad N^{\circ} 34$

Septiembre - Diciembre 2021

Tercera Época

Maracaibo-Veneruela 


\title{
Evaluación de la ecoeficiencia en la municipalidad distrital José Crespo y Castillo, Perú
}

\author{
Joao A. Rosas Ruiz* \\ Ronald H. Puerta Tuesta** \\ Manuel E. Reátegui-Inga*** \\ Reiner P.G. Reátegui Inga **** \\ Eli Morales Rojas *****
}

RESUMEN

El objetivo de esta investigación fue evaluar la ecoeficiencia de la municipalidad distrital José Crespo y Castillo de Perú; para ello se elaboró una línea base y la formulación e implementación de indicadores para el plan de ecoeficiencia. La estandarización de los datos se realizó mediante la aplicación de máximos y mínimos. La ponderación de indicadores se estableció mediante el método Delphi y la validación de instrumentos fue por expertos. La elaboración del biograma se realizó en función a los índices de ecoeficiencia. Se obtuvieron resultados mayores a lo esperado en la etapa de planificación. También se conoció el estado en el que se encuentra la municipalidad distrital José Crespo y Castillo por medio del biograma, el cual resultó no ecoeficiente e inestable.

PALABRAS CLAVE: equilibrio ecológico; presupuesto del Estado; gasto público; administración.

*Investigador del Instituto Municipalidad Distrital de José Crespo y Castillo, Aucayacu, ORCID: https://orcid.org/0000-0001-8517-0866. E-mail: joantnio0466@gmail.com

**Investigador de la Universidad Nacional Agraria de la Selva, Tingo María, Perú. ORCID: https://orcid.org/0000-0001-5777-7855. E-mail: ronald.puerta@unas.edu.pe

** Investigador de la Universidad Nacional de Jaén, Jaén, Perú. ORCID: https://orcid.org/0000-0002-5417-6509. E-mail: manuel.reategui@unj.edu.pe

**** Investigador de la Universidad Nacional Toribio Rodríguez de Mendoza de Amazonas, Chachapoyas, Perú. reiner.reategui@untrm.edu.pe

ORCID: https://orcid.org/0000-0003-1532-991X

*****Investigador del Instituto de Investigación para el Desarrollo Sustentable de Ceja de Selva, Universidad Nacional Toribio Rodríguez de Mendoza de Amazonas. ORCID: https://orcid.org/0000-0002-8623-3192. E-mail: elimor.4740@gmail.com 


\section{Evaluation of eco-efficiency in the district municipality of José Crespo y Castillo, Peru}

ABSTRACT

The objective of this research was to evaluate the eco-efficiency of the district municipality José Crespo y Castillo de Perú; for this, a baseline was drawn up and indicators were formulated and implemented for the eco-efficiency plan. The standardization of the data was carried out by applying maximums and minimums. The weighting of indicators was established using the Delphi method and the instruments were validated by experts. The development of the biogram was carried out based on the eco-efficiency indices. Higher than expected results were obtained in the planning stage. The state in which the district municipality José Crespo y Castillo is located was also known through the biogram, which was not eco-efficient and unstable.

KEYWORDS: Ecological balance; State budget; public expenditure; administration

\section{Introducción}

La ecoeficiencia fue implantada por el Consejo Empresarial Mundial para el Desarrollo (con sus siglas en inglés WBCSD) en 1992, con el fin de generar bienes y servicios utilizando menos materia prima, y de esta forma generar menos residuos sólidos y emisión de gases (Sánchez, 2011). A nivel de Latinoamérica, las estrategias de ecoeficiencia han sido escazas; existen empresas transnacionales como el Banco Interamericano de Desarrollo (BID), que invierten para la aplicación de estas estrategias, lo que involucra contar con recurso financiero y apoyo material para la adquisición de tecnologías limpias y una gestión ambiental adecuada (Merlo y Cuesta, 2018).

En el Perú, los primeros avances en ecoeficiencia se dieron mediante la promulgación de la Ley N²9289 "Ley de Presupuesto del sector público para el año fiscal 2009”, donde se indicaba que se deben tomar acciones ecoeficientes en los recursos de energía, agua, papel y combustibles. Después apareció el Decreto Supremo N009-2009-MINAM [Ministerio del Ambiente], donde se menciona la adopción obligatoria de medidas de ecoeficiencia en las entidades del sector público, así como también las empresas que brindan servicios al Estado (Decreto Supremo N009-2009-MINAM, 2009). 
Sin embargo, las instituciones públicas -en su gran mayoría- aún no implementan lo dispuesto el D.S. N 009 - 2009- MINAM, siendo una de ellas la municipalidad distrital José Crespo y Castillo, que no cuenta con estudios ni medidas en ecoeficiencia (Marrou y García, 2017; Municipalidad Provincial de El Dorado, 2016).

La ecoeficiencia ayuda a reducir costos de producción o servicios, lo que coadyuva en la competitividad, satisfacción de los clientes y eleva la calidad de vida de la población, minimizando los impactos ambientales; consecuentemente, la institución u organización es más rentable, responsable y sustentable (Advíncula et al., 2014).

Además, la ecoeficiencia es una herramienta que permite evaluar el funcionamiento ambiental de diferentes empresas, así como la generación de oportunidades de mejora ambiental (Rincón y Wellens, 2011), y es importante para la aplicación de instrumentos de gestión para el reciclaje y sistemas de eliminación de residuos (Sanguinetti et al., 2019; Marín y Ferrer, 2020).

En base a lo mencionado, el objetivo general de esta investigación fue evaluar la ecoeficiencia en la municipalidad distrital José Crespo y Castillo. En consecuencia, se trazaron los siguientes objetivos específicos: a) determinar los índices de ecoeficiencia; b) proponer un plan de medidas ecoeficientes para la municipalidad distrital José Crespo y Castillo.

1. Materiales y métodos

\section{1.l. Ubicación}

La investigación se desarrolló en la municipalidad distrital José Crespo y Castillo Aucayacu, provincia de Leoncio Prado, región Huánuco, Perú (Figura l).

\subsection{Metodología}

Para la determinación de la línea base se recopiló información correspondiente a un año, desde septiembre del 2015 hasta agosto del 2016. Se tuvo una población de 126 administrativos y se tomó como muestra a 95 para la aplicación de la encuesta. Rigiéndose por las fórmulas respectivas, se promedió el total de meses de la línea base dividida por el promedio de número de colaboradores de ese periodo. Se realizó el registro de indicadores de energía eléctrica, consumo de agua, los cuales fueron obtenidos por medio de las boletas de 
REVISTA DE LA UNIVERSIDAD DEL ZULIA. 3época. Año $12 \mathrm{~N}^{\circ}$ 34, 2021 Joao A. Rosas Ruiz et al. /// Evaluación de la ecoeficiencia en la municipalidad distrital José Crespo... 167-184 DOI: http://dx.doi.org/10.46925//rdluz.34.11

consumo reportadas por las entidades como ELECTROCENTRO S.A y SEDA Huánuco S.A.; el resto de indicadores, como el consumo de combustibles, consumo de útiles de oficina, se obtuvieron a partir de la información brindada por las áreas de tesorería y logística de la municipalidad.

Figura 1. Mapa de ubicación de la municipalidad distrital José Crespo y Castillo
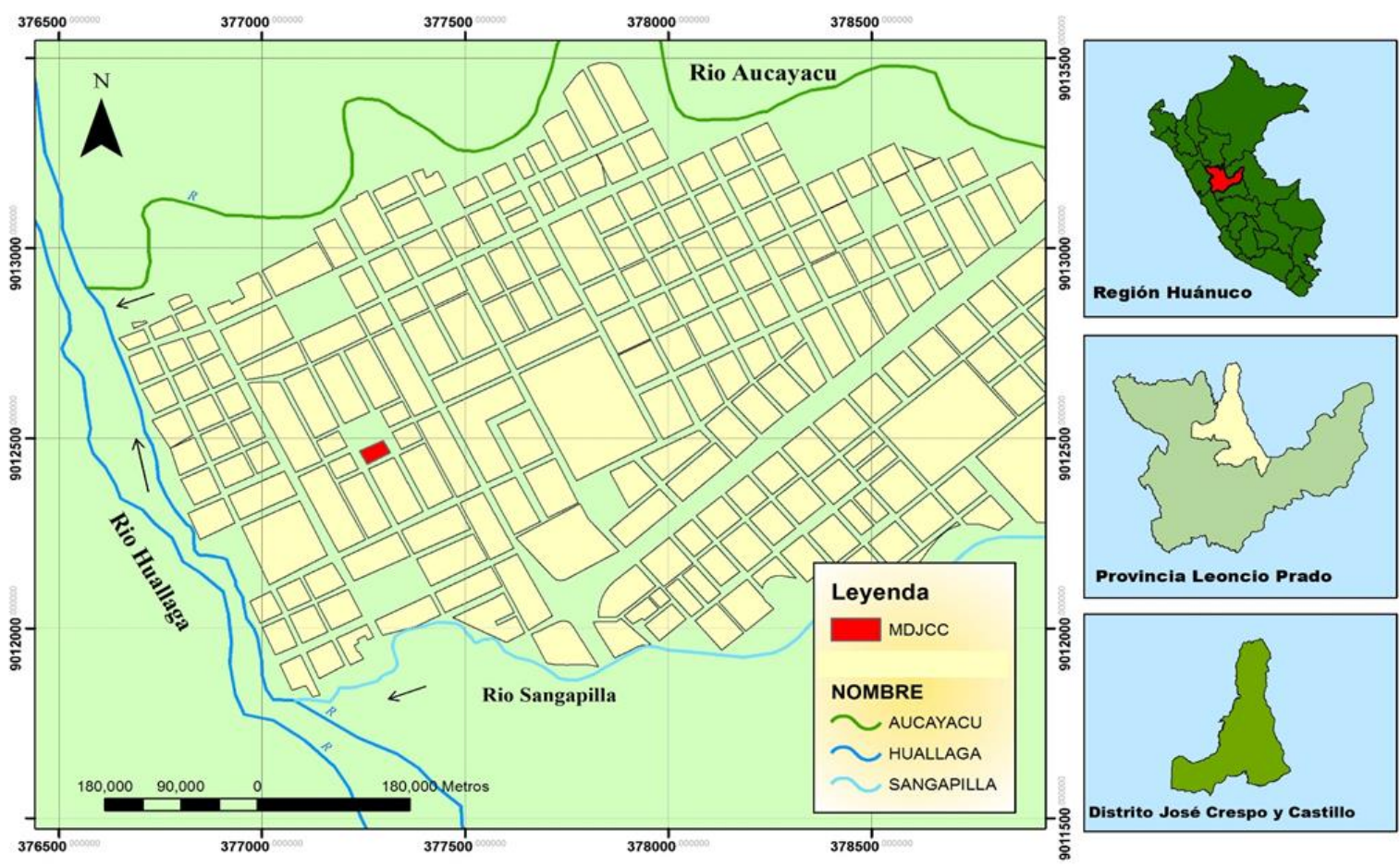

Estandarización de indicadores: Una vez seleccionados los indicadores, se definió su relación positiva o negativa con la eficiencia, para la cual se recurrió al método de máximos y mínimos (Di Pasquale y Balsa, 2017).

Niveles máximos y mínimos: Se calcularon los valores máximos y mínimos de cada indicador mediante la fórmula (1) de mínimos y fórmula (2) de máximos, respectivamente:

$$
\begin{aligned}
& f(X)=(X-m) /(M-m) \\
& f(X)=(X-M) /(m-M)
\end{aligned}
$$

Dónde:

$$
\begin{aligned}
& \mathrm{M}=\text { Es el nivel máximo en el periodo } \\
& \mathrm{m}=\text { Es el nivel mínimo en el periodo }
\end{aligned}
$$

$\mathrm{X}=$ valor correspondiente del indicador para unidad de análisis determinada en el período. 
Mediante la utilización de estas fórmulas se obtuvieron índices individuales para el indicador, los cuales fluctuarán entre 0 y 1 . Donde el valor 0 representa la peor situación del sistema y el valor 1 representa una relación positiva.

Ponderación de indicadores: Para obtener la ponderación de los indicadores de ecoeficiencia, se recurrió al método Delphi, para lo cual se realizaron dos consultas: la primera buscó conocer el grado de influencia de cada indicador a la ecoeficiencia; y la segunda buscó la convergencia de resultados con la finalidad de disminuir la dispersión, precisando la mediana para confirmar sus respuestas (Loor-Carvajal et al., 2020).

Se conformó un panel de expertos de 15 profesionales de diferentes instituciones, con conocimiento en ecoeficiencia, quienes dieron su opinión independiente a la importancia de cada indicador. Para la ponderación de los indicadores de ecoeficiencia se emplearon frecuencias absolutas y relativas acumuladas, con la fórmula (3).

$\mathrm{fr}_{-} \mathrm{i}=\mathrm{f}_{-} \mathrm{i} / \mathrm{n}$

Dónde:

fi = Número de veces de la muestra

$\mathrm{n}=$ Número total de observaciones

La suma de frecuencias relativas tiende a 1. Para interpretar las frecuencias relativas se multiplicó por 100 y se leyó en porcentaje (Levin y Rubin, 2004). Una vez determinados los factores de ponderación (pesos) se procedió a agregar todas las variables o sub indicadores en un indicador sintético. Habiendo estandarizado los indicadores, se ponderaron de acuerdo al nivel de importancia estipulado por el método de Delphi (fórmula 4).

$$
\mathrm{SPE}=\sum_{\mathrm{i}=1}^{\mathrm{n}} \mathrm{M}_{\mathrm{i}} \mathrm{x} \mathrm{P}_{\mathrm{i}}
$$

Dónde:

$\mathrm{Mi}=$ Calidad ambiental del indicador (0 a l)

$\mathrm{Pi}=$ Ponderación del indicador

\subsection{Elaboración del biograma}

Se utilizaron los resultados obtenidos en el cálculo del índice de ecoeficiencia (subíndices de todos los indicadores); estos se agregaron para obtener el índice integrado, 


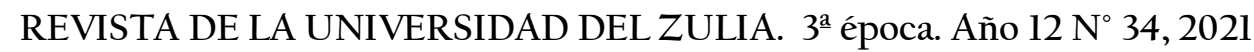
Joao A. Rosas Ruiz et al. /// Evaluación de la ecoeficiencia en la municipalidad distrital José Crespo... 167-184 DOI: http://dx.doi.org/10.46925//rdluz.34.11

fórmula (5) del Índice de Ecoeficiencia, teniendo en cuenta las modificaciones planteadas por Zambrano (2014) y Casado (2014). La agregación consiste en sumar los índices de los indicadores de ecoeficiencia para cada unidad de análisis.

$$
I E=\sum_{i=1}^{n} M_{1} x P_{1}+M_{2} \times P_{2}+\cdots
$$

En el biograma se utilizaron cinco colores para caracterizar fácilmente el estado de desarrollo sostenible de la unidad de análisis (Figura 4).

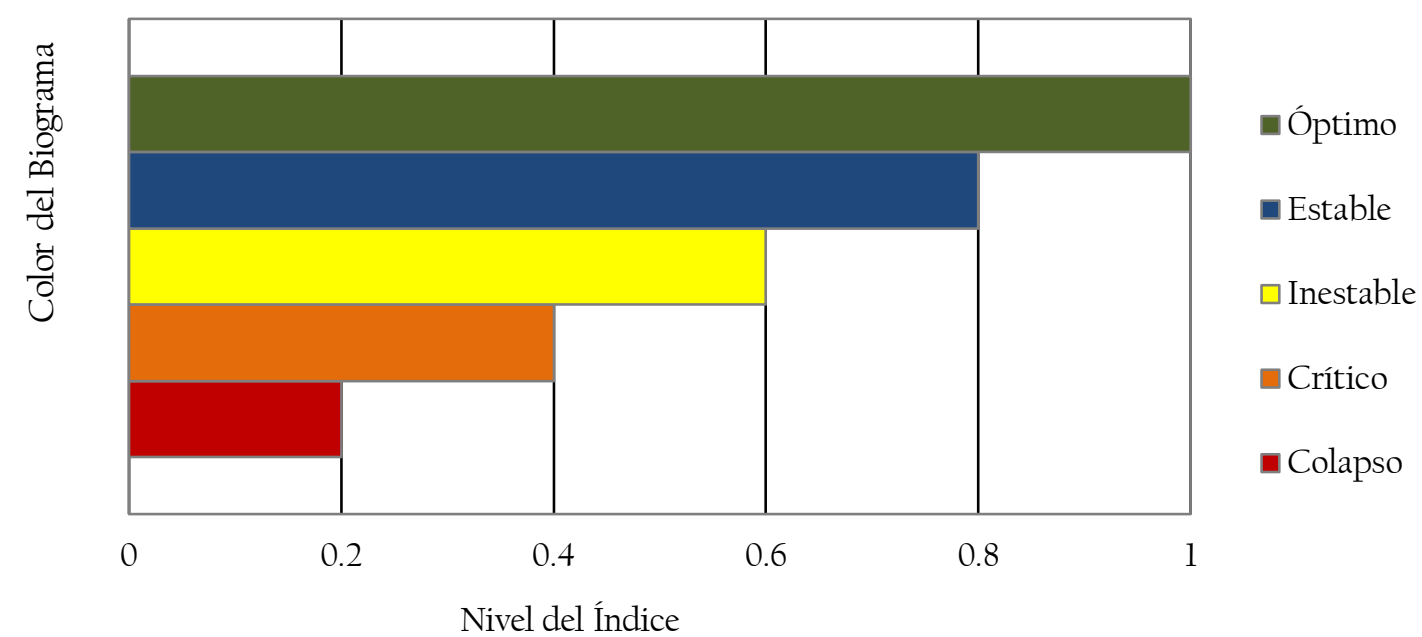

Figura 4. Biograma. Metodología para estimar el nivel de desarrollo sostenible de los territorios rurales (Sepúlveda, 2008).

\subsection{Implementación del plan de medidas de ecoeficiencia}

Para la etapa de implementación se contemplaron los planes de medidas de ecoeficiencia que fueron viables para cada indicador desarrollado en el diagnóstico, teniendo en cuenta la priorización de ellos.

Las oportunidades de mejora: Se describieron aquellas prácticas que son poco o nada ecoeficientes y que han sido identificadas dentro de la municipalidad.

Medidas ecoeficientes a implementar: Se describieron aquellas prácticas, mantenimiento o mejora tecnológica que deben realizarse dentro de la municipalidad, con la finalidad de cumplir con lo dispuesto en el D.S Nº09-2009-MINAM y su modificatoria D.S Nºll-2010-MINAM-Medidas de Ecoeficiencia para el Sector Público (MINAM, 2010). 
Inversión en la medida ecoeficiente implementada: Se consideró el monto económico al cual asciende la implementación de las buenas prácticas, el mantenimiento o mejora tecnológica, expresado en soles.

Monitoreo del programa de ecoeficiencia: El monitoreo de los indicadores de desempeño se llevó a cabo según los criterios establecidos en la fase preparatoria de la implementación y el impacto de las medidas; se evaluó comparando los valores de los indicadores obtenidos a través del monitoreo, con los valores de referencia (indicadores medidos antes de la implementación de las medidas de ecoeficiencia).

Después de haber realizado la línea base de ecoeficiencia, se plantearon capacitaciones ambientales para las áreas administrativas en temas relacionadas a buenas prácticas ambientales y ecoeficiencia, de tal manera que los colaboradores tengan conocimientos, se motiven y adquieran compromiso con su municipalidad. Previamente se aplicó una evaluación de entrada y al culminar dicha capacitación se tomó una evaluación de salida, con la finalidad de ver si hubo un incremento en cuanto al conocimiento de datos.

Determinación de la efectividad de costos de las medidas ecoeficientes implementadas en la municipalidad distrital José Crespo y Castillo: Para la determinación de la relación costo/efectividad se tuvo en consideración la inversión realizada por la institución en los programas implementados; estos programas incluyen medidas ecoeficientes que fueron prioritarias.

\subsection{Relación costo - efectividad}

Es el indicador de decisión que muestra los resultados obtenidos por cada programa implementado (efectividad) y la inversión realizada por la institución en dicho programa (costo), que se obtuvo mediante la fórmula (6) de relación costo efectividad de las medidas ecoeficientes implementadas.

$$
\mathrm{RCE}=\frac{\text { Resultados obtenidos por medida implementada }(\%)}{\text { Costo de implementacion de la medida ecoeficiente(soles) }}
$$

Dónde:

RCE = Relación costo/efectividad de la medida implementada 


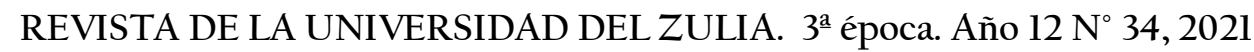

Joao A. Rosas Ruiz et al. /// Evaluación de la ecoeficiencia en la municipalidad distrital José Crespo... 167-184

DOI: http://dx.doi.org/10.46925//rdluz.34.11

2. Resultados

2.1. Determinación de la línea base

En el Cuadro 1 se muestran los indicadores de desempeño para cada indicador determinado en la línea base.

Cuadro 1. Indicadores de desempeño de la línea base

\begin{tabular}{|c|c|c|c|c|}
\hline Indicador & Sub indicador & Código & Unidad & Índice de desempeño \\
\hline $\begin{array}{c}\text { Consumo de } \\
\text { energía eléctrica }\end{array}$ & Energía eléctrica & IDE & kWh/colaborador & 50,14 \\
\hline \multirow{2}{*}{$\begin{array}{l}\text { Consumo de } \\
\text { combustibles }\end{array}$} & Diésel & \multirow{2}{*}{ IDC } & \multirow{2}{*}{ Gal/vehículo } & 330,24 \\
\hline & Gasolina 90 & & & 22,85 \\
\hline Consumo de agua & Agua & IDA & m3/colaborador & 4,60 \\
\hline \multirow{3}{*}{$\begin{array}{l}\text { Consumo de útiles } \\
\text { de oficina }\end{array}$} & Papel Bond & \multirow{3}{*}{ IDU } & \multirow[b]{2}{*}{$\mathrm{kg} /$ colaborador } & 2,54 \\
\hline & $\begin{array}{c}\text { Otros papeles y } \\
\text { sobres }\end{array}$ & & & 0,11 \\
\hline & $\begin{array}{l}\text { Cartuchos y } \\
\text { Tóneres }\end{array}$ & & Unidad/colaborador & 0,20 \\
\hline \multirow{8}{*}{$\begin{array}{l}\text { Generación de } \\
\text { residuos sólidos }\end{array}$} & Papeles & \multirow{8}{*}{ IDRS } & \multirow{8}{*}{$\mathrm{kg} /$ colaborador } & \multirow{8}{*}{1,29} \\
\hline & Cartones & & & \\
\hline & Plásticos & & & \\
\hline & Vidrios & & & \\
\hline & Tóneres & & & \\
\hline & Metales & & & \\
\hline & Orgánicos & & & \\
\hline & Otros & & & \\
\hline \multirow{5}{*}{$\begin{array}{l}\text { Prácticas contrarias } \\
\text { a la ecoeficiencia }\end{array}$} & Energía eléctrica & \multirow{5}{*}{ IPL } & \multirow{5}{*}{$\%$} & \multirow{5}{*}{59,018} \\
\hline & Combustible & & & \\
\hline & Agua & & & \\
\hline & Útiles de oficina & & & \\
\hline & Residuos sólidos & & & \\
\hline
\end{tabular}

2.2. Índice de ecoeficiencia en la municipalidad distrital José Crespo y Castillo 


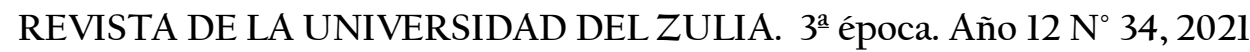
Joao A. Rosas Ruiz et al. /// Evaluación de la ecoeficiencia en la municipalidad distrital José Crespo... 167-184

DOI: http://dx.doi.org/10.46925//rdluz.34.11

\begin{tabular}{|c|c|c|c|c|c|c|c|}
\hline Código & Indicadores & $\begin{array}{c}\text { Sub } \\
\text { indicador }\end{array}$ & $\begin{array}{c}\text { Sub } \\
\text { índice }\end{array}$ & FP & SP & $\begin{array}{l}\text { Total } \\
\text { etapa }\end{array}$ & Índice \\
\hline IDE & \multicolumn{2}{|c|}{ Consumo de energía eléctrica } & 0,36 & 0,18 & 0,07 & 0,07 & \\
\hline \multirow{2}{*}{ IDC } & Consumo de & Diésel & \multirow{2}{*}{0,40} & 0,17 & 0,06 & \multirow{2}{*}{0,07} & \\
\hline & combustibles & Gasolina 90 & & 0,17 & 0,07 & & \\
\hline IDA & Consum & de agua & 0,65 & 0,18 & 0,11 & 0,11 & \\
\hline \multirow{3}{*}{ IDU } & \multirow{3}{*}{$\begin{array}{c}\text { Consumo de útiles } \\
\text { de oficina }\end{array}$} & Papel bond & & 0,15 & 0,04 & \multirow{3}{*}{0,07} & \\
\hline & & $\begin{array}{c}\text { Otros papeles y } \\
\text { sobres }\end{array}$ & 0,46 & 0,15 & 0,08 & & \\
\hline & & Cartuchos y tóners & & 0,15 & 0,09 & & \\
\hline \multirow{8}{*}{ IDRS } & \multirow{8}{*}{$\begin{array}{l}\text { Generación de } \\
\text { residuos sólidos }\end{array}$} & Papeles & \multirow{8}{*}{0,53} & 0,17 & 0,13 & \multirow{8}{*}{0,09} & \\
\hline & & Cartones & & 0,17 & 0,13 & & \\
\hline & & Plásticos & & 0,17 & 0,05 & & 0,49 \\
\hline & & Vidrios & & 0,17 & 0,16 & & \\
\hline & & Tóners & & 0,17 & 0,12 & & \\
\hline & & Metales & & 0,17 & 0,00 & & \\
\hline & & Orgánicos & & 0,17 & 0,11 & & \\
\hline & & Otros & & 0,17 & 0,00 & & \\
\hline \multirow{5}{*}{ IDPL } & \multirow{5}{*}{$\begin{array}{c}\text { Prácticas contrarias } \\
\text { a la ecoeficiencia }\end{array}$} & Energía eléctrica & \multirow{5}{*}{0,41} & 0,16 & 0,06 & \multirow{5}{*}{0,07} & \\
\hline & & Combustible & & 0.16 & 0,08 & & \\
\hline & & Agua & & 0,16 & 0,05 & & \\
\hline & & Útiles de oficina & & 0,16 & 0,10 & & \\
\hline & & Residuos Sólidos & & 0,16 & 0,04 & & \\
\hline
\end{tabular}

Tabla 2. Cálculo del índice de ecoeficiencia

FP=Factor de ponderación; $\mathrm{SP}=$ Subíndice ponderado

El índice de ecoeficiencia fue de 0,49, lo que permite clasificar a la municipalidad distrital José Crespo y Castillo como no eficiente y encontrándose en niveles inestables en el uso de sus recursos (Tabla 2). 


\subsection{Estado del sistema de la municipalidad distrital José Crespo y Castillo}

Los resultados arrojaron que el índice fue de 0,49, valor que se encontró en el intervalo 0,4-0,6, que corresponde a un sistema inestable, de color amarillo (Sepúlveda, 2008).

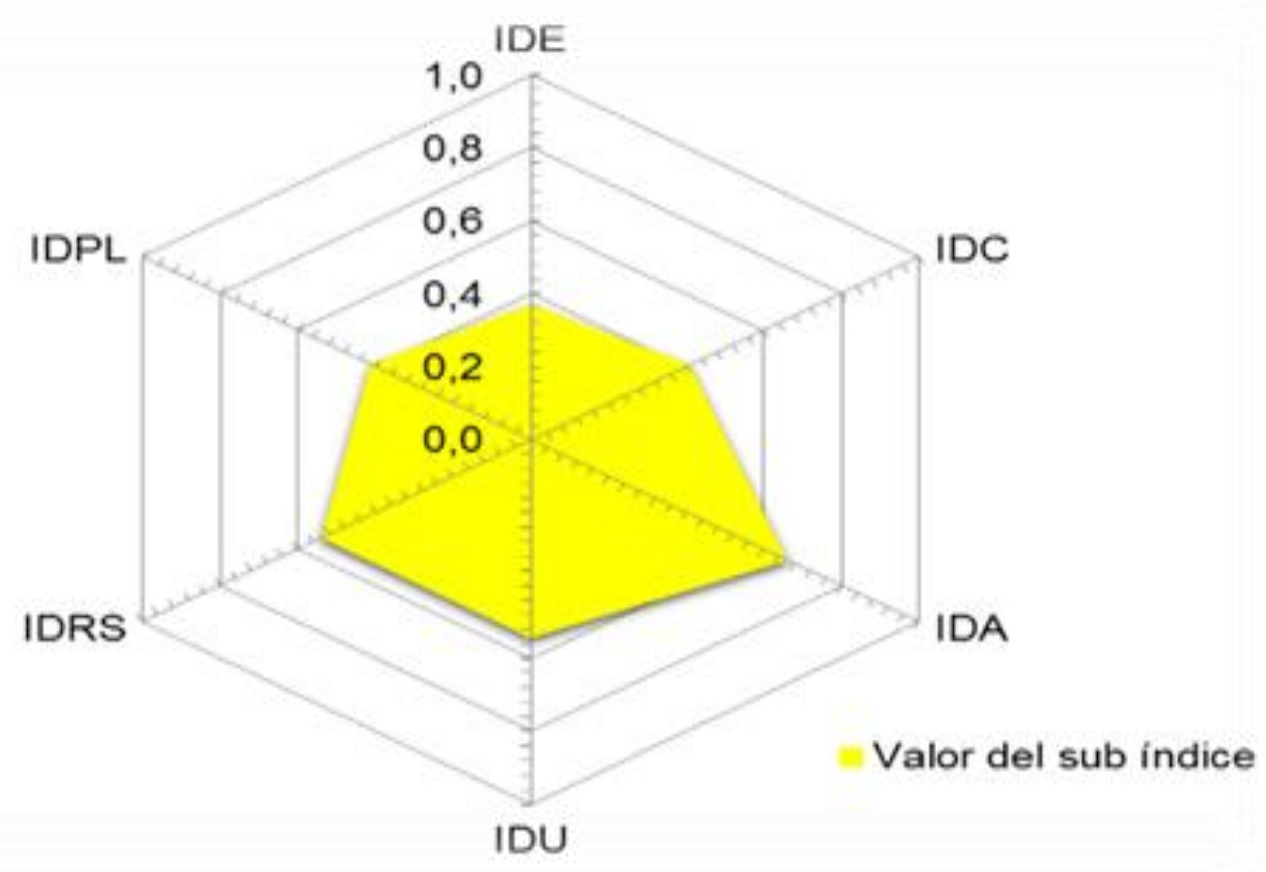

Figura 2. Biograma construido con valores de los subíndices

2.4. Plan de medidas de ecoeficiencia en la municipalidad distrital José Crespo y Castillo

En el Cuadro 3 se muestra la medida ecoeficiente propuesta y los costos de inversión por la municipalidad. Se encontró que la asignación económica a estas medidas (buenas prácticas y mantenimiento) fue de cero soles; esto se debe a que los gastos fueron asumidos por los investigadores. 
REVISTA DE LA UNIVERSIDAD DEL ZULIA. 3a época. Año $12 \mathrm{~N}^{\circ} 34,2021$

Joao A. Rosas Ruiz et al. /// Evaluación de la ecoeficiencia en la municipalidad distrital José Crespo... 167-184

DOI: http://dx.doi.org/10.46925//rdluz.34.11

Cuadro 3. Plan de medidas de ecoeficiencia

\begin{tabular}{|c|c|c|c|c|}
\hline $\mathrm{N}^{\circ}$ & Medida de ecoeficiencia implementada & Priorización & Inversión (S/) & Avance \\
\hline \multicolumn{5}{|c|}{ Energía eléctrica } \\
\hline 1 & $\begin{array}{l}\text { Apagar los equipos electrónicos cuando no se tenga } \\
\text { prevista su inmediata utilización }\end{array}$ & \multirow{4}{*}{ Alta } & \multirow{4}{*}{0} & \multirow{4}{*}{ Alta } \\
\hline 2 & $\begin{array}{l}\text { Programar pantallas de las computadoras en modo de } \\
\text { ahorro de energía, que permita apagar } \\
\text { automáticamente el monitor sin perder información y } \\
\text { al movimiento del mouse se vuelva a activar. }\end{array}$ & & & \\
\hline 3 & Usar el aire acondicionado con las ventanas cerradas. & & & \\
\hline 4 & Charlas al personal sobre eficiencia energética. & & & \\
\hline \multicolumn{5}{|c|}{ Combustible } \\
\hline 5 & $\begin{array}{l}\text { Estabilización en el consumo de gasolina de } 90 \text { y diésel } \\
2\end{array}$ & \multirow{2}{*}{ Alta } & \multirow{2}{*}{0} & \multirow{2}{*}{ Alta } \\
\hline 6 & $\begin{array}{llll}\begin{array}{l}\text { Programar mantenimientos } \\
\text { preventivas. }\end{array} & \text { y supervisiones } \\
\end{array}$ & & & \\
\hline
\end{tabular}

Consumo de agua

$7 \quad$ Reducción del consumo de agua

8 Charlas al personal sobre uso y ahorro del agua

Alta $\quad 0 \quad$ Alta

Útiles de oficina

\begin{tabular}{|c|c|c|c|c|}
\hline 9 & $\begin{array}{l}\text { Charlas informativas sobre el uso racional de útiles de } \\
\text { oficina. }\end{array}$ & \multirow{6}{*}{ Alta } & \multirow{6}{*}{0} & \multirow{6}{*}{ Alta } \\
\hline 10 & $\begin{array}{l}\text { Asignar a cada trabajador una cantidad máxima de } \\
\text { impresiones y fotocopias. }\end{array}$ & & & \\
\hline 10 & $\begin{array}{l}\text { Racionalización de útiles de oficinas a las diferentes } \\
\text { áreas }\end{array}$ & & & \\
\hline 11 & $\begin{array}{l}\text { Reutilizar las hojas impresas por una cara y que no } \\
\text { tienen valor, como hojas de borrador. }\end{array}$ & & & \\
\hline 12 & $\begin{array}{l}\text { Instruir al personal en revisar los documentos en forma } \\
\text { virtual con el uso de opción control de cambios. }\end{array}$ & & & \\
\hline 13 & $\begin{array}{l}\text { Utilizar el modo "borrador" en la impresión de los } \\
\text { documentos de trabajo que sea indispensable imprimir. }\end{array}$ & & & \\
\hline
\end{tabular}


Generación de residuos sólidos

Difundir los alcances del programa de segregación y
elaborar charlas informativas.

15 Sensibilizar a los colaboradores en temas de manejo de residuos sólidos.

16 Capacitación de todo el personal en temas de

Alta

0

Alta

16 ecoeficiencia y manejo de residuos sólidos.

17 Gestión de residuos con propósitos de venta

2.5. Resultados de la implementación de las actividades de educación ambiental para los colaboradores de la municipalidad distrital José Crespo y Castillo

Los resultados (figura 3) indicaron un incremento de las notas en la prueba de salida representada por la línea amarilla, donde en su mayoría fueron aprobatorias, en comparación a la prueba de entrada donde las notas fueron desaprobatorias, representadas por la línea roja. La cantidad de asistentes a la capacitación fue de 29 colaboradores municipales, en su mayoría personal nombrado de la institución.
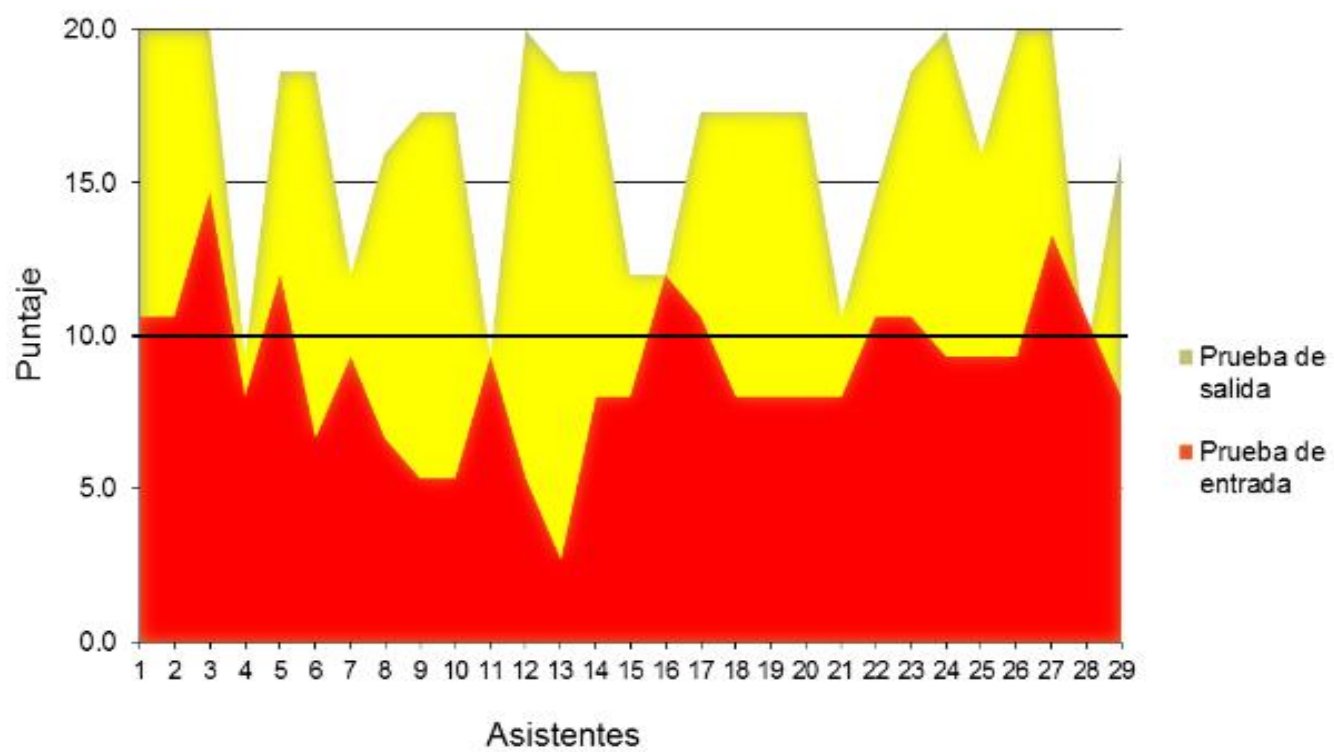

Figura 3. Resultados de la prueba de entrada y salida 
REVISTA DE LA UNIVERSIDAD DEL ZULIA. 3a época. Año $12 \mathrm{~N}^{\circ} 34,2021$

Joao A. Rosas Ruiz et al. /// Evaluación de la ecoeficiencia en la municipalidad distrital José Crespo... 167-184

DOI: http://dx.doi.org/10.46925//rdluz.34.11

En la figura 4 se presentan los objetivos de los costos implementados, así como la efectividad obtenida después de 5 meses de haberse implementado el plan de ecoeficiencia.

Figura 4. Efectividad de los programas implementados

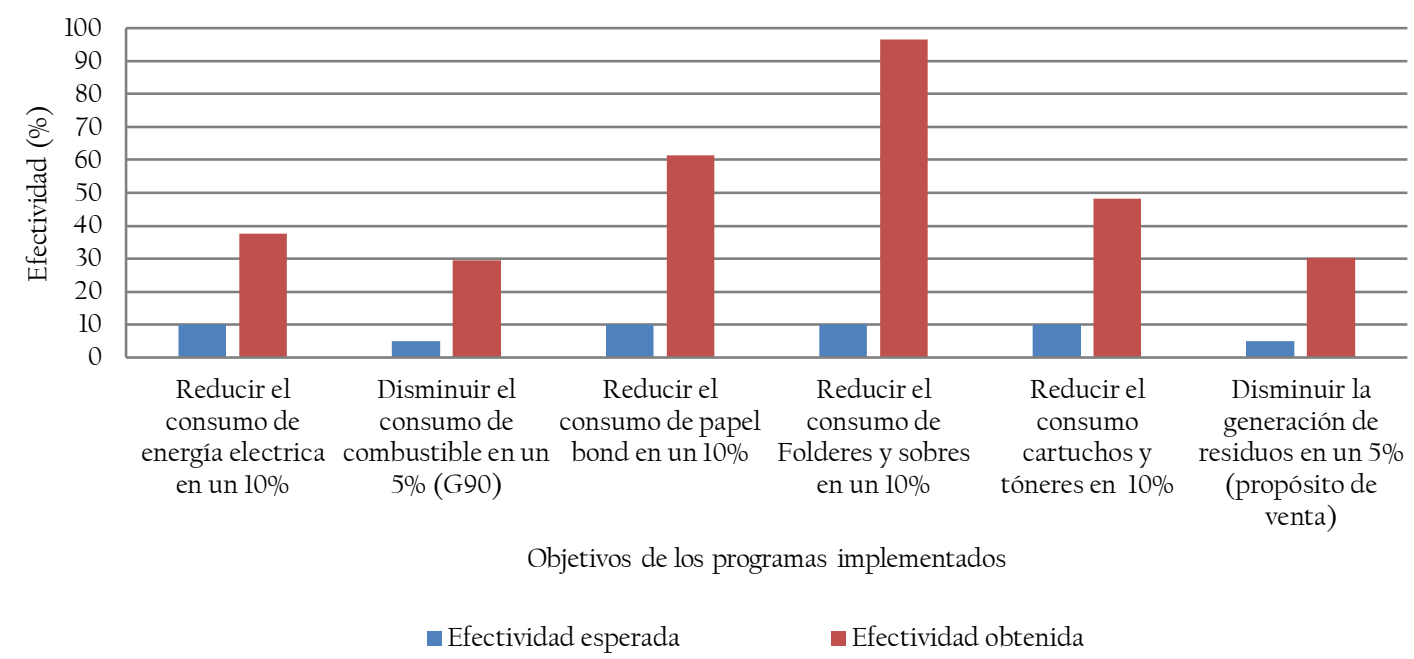

La Figura 5 muestra que la implementación del plan de ecoeficiencia generó ahorros superiores a los planteados en la etapa de formulación del plan de ecoeficiencia.

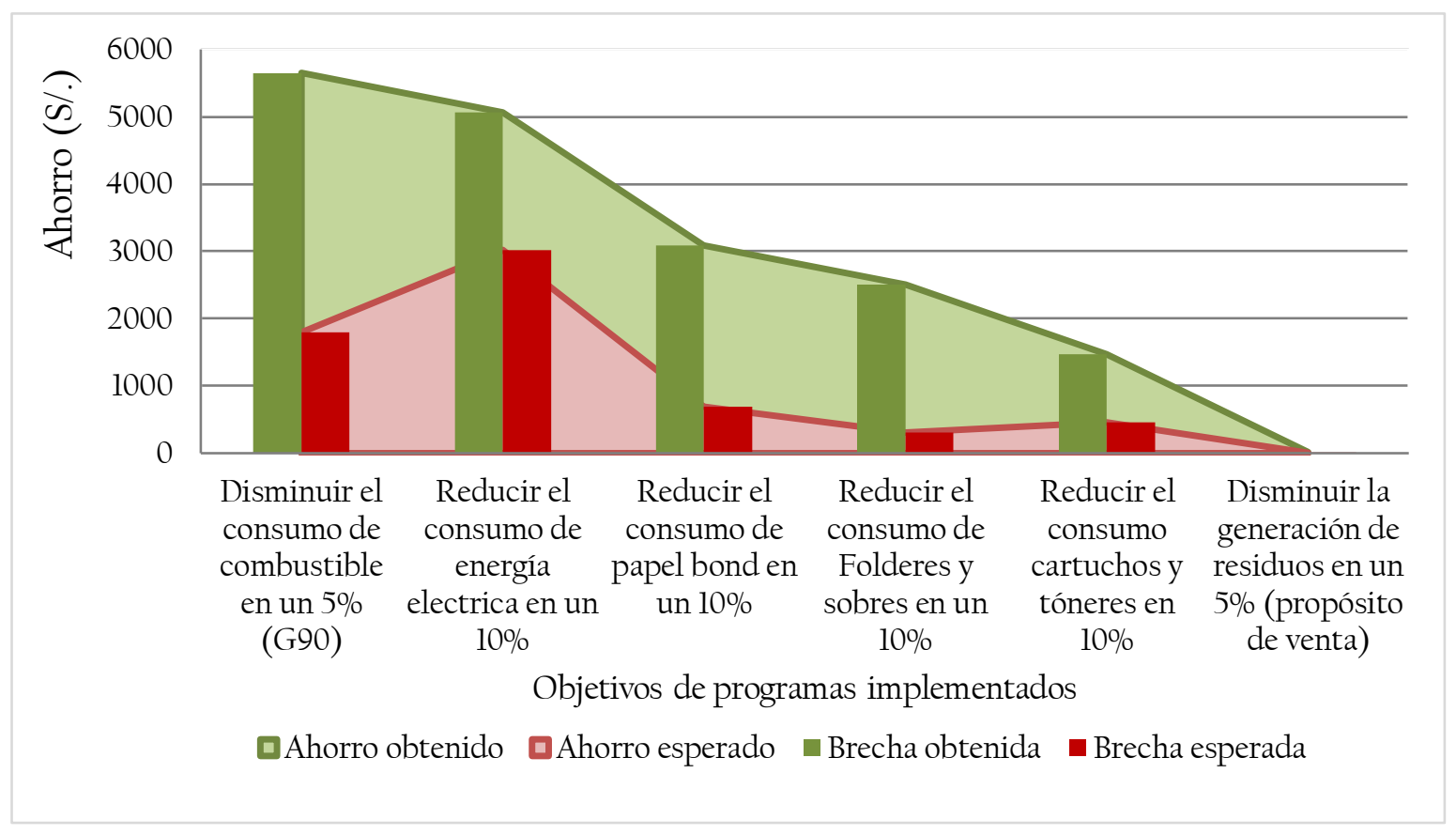

Figura 5. Ahorros generados por el plan de ecoeficiencia, 2015 - 2016 


\section{Discusión}

El desempeño del consumo de energía eléctrica fue de 50,14 kWh/colaborador, el cual es muy inferior a lo reportado por el Ministerio del Ambiente (MINAM, 2016) en su informe anual de instituciones ecoeficientes de 1039,56 kWh/colaborador. El plan de ecoeficiencia de la municipalidad provincial de Leoncio Prado reportó para este indicador un costo promedio de S/ 10 438,40 mensuales (Municipalidad Provincial de Leoncio Prado [MPLP], 2017), en comparación con lo obtenido en la línea base para la municipalidad distrital de José Crespo y Castillo, que pagó por este servicio un monto promedio de S/ 4 762,90. La emisión de $\mathrm{CO}_{2}$ equivalente, estuvo de manera indirecta con el consumo de energía eléctrica; este reporte nos permite conocer cuánto de este gas de efecto invernadero se emite a la atmósfera (una de las políticas de calidad ambiental que busca el Ministerio del Ambiente es reducir la contaminación ambiental según lo estipulado en el Decreto Supremo N009-2009 - MINAM). Lo que se busca con la obtención de este dato es generar políticas educativas dentro de la institución para así fomentar conocimientos y actitudes en temas de consumo responsable de energía.

El indicador de desempeño de agua (4,60 m³/colaborador) es inferior a lo presentado en el informe de instituciones públicas ecoeficientes, cuyo valor es $34,43 \mathrm{~m}^{3}$ por colaborador (MINAM, 2016). La municipalidad distrital José Crespo y Castillo paga por el servicio de agua potable la suma promedio por mes de S/ 535,25, a diferencia de la Municipalidad Provincial de Leoncio Prado cuya suma por pago de este servicio es de S/ 2 904,25 mensualmente. Cabe señalar que la cantidad de personal en la municipalidad de José Crespo y Castillo es menor, pues solo cuenta con 95 colaboradores a diferencia de Leoncio Prado que cuenta con 349 colaboradores (MPLP, 2017). Los resultados obtenidos para el indicador de desempeño en el consumo de útiles de oficina, muestran que la municipalidad distrital José Crespo y Castillo mensualmente gasta alrededor de 52,27 millares de papel bond, 19,33 tintas de impresión y tóneres, a diferencia de la provincia cuyos valores para el consumo de útiles de escritorio son 58 millares de papel bond, 12 tintas para impresora y 34 tóneres, detallados en su plan de ecoeficiencia para el año 2016. En este sentido, es importante el desarrollo de planes e indicadores de consumo en las entidades (Heras-Saizarbitoria et al., 2020). 
Los indicadores que necesitaron priorización fueron los de combustible, útiles de escritorio y generación de residuos sólidos, los cuales obtuvieron un mayor porcentaje en sus respuestas negativas, a diferencia del indicador consumo de agua que dentro del conjunto de indicadores planteados se encontró estable, siendo la priorización en temas de buenas prácticas de consumo de agua complementarias o de carácter informativo. El valor del índice, luego de la ponderación, resultó igual a 0,49; este valor es inferior al obtenido por Casado (2014) (0,5l), pero permaneciendo en el mismo intervalo clasificado como un estado inestable, según Sepúlveda (2008). El ahorro de agua en las instituciones es de suma importancia y debe ser una prioridad hacia la integración del ciclo de vida (Lam et al., 2017).

Las oportunidades de mejora guardan relación con el manejo de recursos por parte de la institución; la elaboración y ejecución del plan de ecoeficiencia incrementará la competitividad de la institución y se reducirá los impactos al ambiente (MINAM, 2016). Estas oportunidades forman parte del proceso de la mejora continua, generando estrategias dentro de las instituciones con la finalidad de cumplir actividades ecoeficientes (Fagnani \& Guimarães, 2017).

El reemplazo de equipos energéticos dentro de la institución, también considerada como medida ecológica, fue propuesto en el marco del cumplimiento del Decreto Supremo Nº042016-EM, que establece el cambio de equipos eléctricos por aquellos con un menor consumo. La municipalidad distrital José Crespo y Castillo cuenta en su mayoría con equipos energéticos ahorradores que se encuentran en óptimas condiciones. Las actividades de educación ambiental fueron implementadas con la finalidad de que los colaboradores alcancen una concepción del medio ambiente y del desarrollo sostenible (Márquez et al., 2021).

Los mayores valores en la relación costo - efectividad se encontraron en el programa de reducción del consumo de útiles de oficina. Todos los programas implementados tuvieron la misma inversión (S/500,00); el ahorro generado en 5 meses desde la implementación del plan fue de S/17 795,17 logrados con los programas implementados, siendo el programa de reducción en el consumo de útiles de escritorio el que presentó un mayor ahorro, menor tiempo en el retorno de la inversión y una mayor relación costo - efectividad.

\section{Conclusiones}


La línea base de ecoeficiencia para la municipalidad distrital José Crespo y Castillo permitió la elaboración del índice de ecoeficiencia con un valor de 0,49, determinándose que la municipalidad de José Crespo y Castillo no es ecoeficiente. Así mismo, el biograma para la representación gráfica del índice de ecoeficiencia indicó que es un sistema inestable. Con la capacitación ambiental a los colaboradores, se logró disminuir el consumo de recursos lográndose ahorrar S/ 17 795,14. La relación costo - efectividad demuestra que la implementación de un plan de medidas de ecoeficiencia no solo brinda una disminución en el consumo de recursos, sino también influye de manera directa el disminuir costos directos y convertirlos en ahorro.

\section{Referencias}

Advíncula, O., García, S., García, J., Toribio, K., y Meza, V. (2014). Plan de ecoeficiencia en el uso del agua potable y análisis de su calidad en las áreas académicas y administrativas de la Universidad Nacional Agraria La Molina. Ecología aplicada, 13(1), 43-55. http://www.scielo.org.pe/scielo.php?pid=S1726-22162014000100005\&script=sci_arttext

Casado, P. (2014). Índice de ecoeficiencia en 5 municipalidades distritales de Leoncio Prado. [Tesis de pregrado]. Universidad Nacional Agraria de la Selva.

Decreto Supremo. N009-2009-MINAM. Medidas de ecoeficiencia para el sector público. https://www.minam.gob.pe/wp-content/uploads/2013/09/ds_009-2009-minam.pdf.

Di Pasquale, E. y Balsa, J. (2017). La técnica de escalamiento lineal por intervalos: una propuesta de estandarización aplicada a la medición de niveles de bienestar social. Revista de Métodos Cuantitativos para la Economía y la Empresa, 23, 164-196. https://www.redalyc.org/pdf/2331/233151826008.pdf

Fagnani, E., \& Guimarães, J. R. (2017). Waste management plan for higher education institutions in developing countries: The Continuous Improvement Cycle model. Journal of Cleaner Production, 147, 108-118. https://doi.org/10.1016/j.jclepro.2017.01.080

Heras-Saizarbitoria, I., García, M., Boiral, O., \& Díaz de Junguitu, A. (2020). The use of ecoefficiency indicators by environmental frontrunner companies. Ecological Indicators, 115(April), 10645l. https://doi.org/10.1016/j.ecolind.2020.106451

Levin, R. y Rubin, D. (2004). Estadística para administración y economía (7ma ed.). Cámara Nacional de la Industria Editorial Mexicana. https://books.google.es/books?hl=es\&lr=\&\&id=uPhtNCqC4isC\&oi=fnd\&pg=PRl3\&\&dq=Levin, +R 
.+2004.+Estad\%C3\%ADstica+para+la+administraci\%C3\%B3n+y+econom\%C3\%ADa.+7+ed.+ M\%C3\%A9xico.+Pearson+educaci\%C3\%B3n.\&ots=Y5CLyrDNpn\&sig=ZqRHBM2WYn6Cgs0 2FmBGylZrSBA $\# \mathrm{v}=$ onepage $\& q \& \&=$ falseLoor-

Loor-Carvajal, G., Rezabala-Encalada, Y., Sánchez-Briones, Y. y Pozo-Rodríguez, J. (2020). El método Delphi: Una aproximación a su utilización en la evaluación del desempeño en Ecuador. $\begin{array}{llll}\text { Dominio de las } & \text { Ciencias, } & 6(4), & \text { 1462-1483. }\end{array}$ https://www.dominiodelasciencias.com/ojs/index.php/es/article/view/1560

Lam, C. M., Leng, L., Chen, P. C., Lee, P. H., \& Hsu, S. C. (2017). Eco-efficiency analysis of nonpotable water systems in domestic buildings. Applied Energy, 202, 293-307. https://doi.org/10.1016/j.apenergy.2017.05.095

Marín L., J., \& Ferrer, L. (2020). Basura marina en la costa Oeste de la Bahía de Amuay, estado Falcón (Venezuela). Revista Latinoamericana De Difusión Científica, 2(2), 6-21. https://doi.org/10.38186/difcie.22.03

Márquez, D., Hernández, A., Márquez, L. y Casas, M. (2021). La educación ambiental: evolución conceptual y metodológica hacia los objetivos del desarrollo sostenible. Revista Universidad y Sociedad, 13(2), 301-310. http://scielo.sld.cu/scielo.php?script=sci_arttext\&pid=S221836202021000200301

Marrou, A. y García, V. (2017). La motivación y la Ecoeficiencia en la Municipalidad de Jesús Lima 2014 [Tesis de posgrado, Universidad César Vallejo] Repositorio institucional. https://repositorio.ucv.edu.pe/handle/20.500.12692/6566

Merlo, O. y Cuesta, P. (2018). Prácticas ecoeficientes en las empresas hoteleras de la ciudad de $\begin{array}{llll}\text { Ibarra-Ecuador. Revista UNIANDES } & \text { Episteme, }\end{array}$ https://dialnet.unirioja.es/servlet/articulo?codigo $=6756404$

Ministerio del Ambiente - MINAM. (2010). Decreto Supremo 011-2010-MINAM: Modifican artículos del Decreto Supremo № 009-2009-MINAM - Medidas de Ecoeficiencia para el Sector Público. https://www.minam.gob.pe/wp-content/uploads/2013/09/ds_011-2010-minam.pdf

Ministerio del Ambiente - MINAM, (2016). Instituciones públicas ecoeficientes: informa anual 2015. https://www.minam.gob.pe/calidadambiental/wpcontent/uploads/sites/22/2017/04/informe_Ecoeficiencia_paginas.compressed.pdf

Municipalidad Provincial de El Dorado. (2016). Línea base de ecoeficiencia de la Municipalidad Provincial de El Dorado. http://sial.minam.gob.pe/eldorado/download/file/fid/62958

Municipalidad Provincial de Leoncio Prado - MPLP. (2017). Directiva Nº02-2017-MPLPDirectiva de medidas de ecoeficiencia en la Municipalidad Provincial de Leoncio Prado. 
https://munitingomaria.gob.pe/mplp/sites/default/files//DIRECTIVA\%20DE\%20MEDIDAS\%2 0DE\%20ECOEFICIENCIA\%20EN\%20LA\%20MPLP\%202017.pdf.

Rincón, E. y Wellens, A. (2011). Cálculo de indicadores de ecoeficiencia para dos empresas ladrilleras Mexicanas. Revista Internacional de Contaminación Ambiental, 27(4), 333-345. http://www.scielo.org.mx/scielo.php?script=sci_arttext\&pid=S0188-49992011000400006

Sanguinetti, C. M. M., Camacho, C. R., Meléndez, M. M., \& Balic, G. C. (2019). Urbanización de viviendas y gestión ecoeficiente de residuos de construcción en Chile: aplicación del modelo español. Ambiente Construido, 19(3), 275-294. https://doi.org/10.1590/s167886212019000300338

Sánchez, Y. (2011). Ecoeficiencia en la universidad hacia un desarrollo sostenible. Gestión en el tercer milenio, 14(27), 47-53. https://revistasinvestigacion.unmsm.edu.pe/index.php/administrativas/article/view/8855

Sepúlveda, S. (2008). Gestión del desarrollo sostenible en territorios rurales: métodos para la planificación.

https://books.google.es/books?hl=es\&lr=\&\&id=2SFHAlHGYekC\&oi=fnd\&pg=PRl7\&dq=metodo logia+para+estimar+el+nivel+de+desarrollo+sostenible+\&ots=kNLkNeCx8C\&sig=3kR4eOId WuDzUIYe2fQDIlbjUko\#v=onepage\&q=metodologia\%20para\%20estimar\%20el\%20nivel\%20 de\%20desarrollo\%20sostenible\&f=false

Zambrano, K. (2014). Ecoeficiencia en instituciones educativas de gestión pública y privada del distrito de Rupa Rupa (Huánuco, Perú). RevIA, 4 (l y 2), 24-29. https://revistas.unas.edu.pe/index.php/revia/article/viewFile/64/5l 